\title{
Research Article \\ Bounding the Output Error in a Buck Power Converter Using Perturbation Theory
}

\author{
Fabiola Angulo, ${ }^{1}$ Enric Fossas, ${ }^{2}$ Tere M. Seara, $^{3}$ and Gerard Olivar ${ }^{1}$ \\ ${ }^{1}$ Departamento de Ingeniería Eléctrica, Electronica y Computación, \\ Universidad Nacional de Colombia, Cra 27 No. 64-60 Manizales, Caldas, Colombia \\ ${ }^{2}$ Institute of Industrial and Control Engineering, Technical University of Catalonia, \\ 08028 Barcelona, Spain \\ ${ }^{3}$ Departamento de Matemàtica Aplicada IV, Universitat Politècnica de Catalunya, \\ 08034 Barcelona, Spain
}

Correspondence should be addressed to Gerard Olivar, golivart@unal.edu.co

Received 20 January 2008; Revised 30 May 2008; Accepted 20 July 2008

Recommended by Oleg Gendelman

We show the main results obtained when applying the average theory to Zero Average Dynamic control technique in a buck power converter with pulse-width modulation (PWM). In particular, we have obtained the bound values for output error and sliding surface. The PWM with centered and lateral pulse configurations were analyzed. The analytical results have confirmed the numerical and experimental results already obtained in previous publications. Moreover, through an important lemma, we have generalized the theory for any stable second-order system with relative degree 2 , using properties related to transformations and stability of linear systems.

Copyright (c) 2008 Fabiola Angulo et al. This is an open access article distributed under the Creative Commons Attribution License, which permits unrestricted use, distribution, and reproduction in any medium, provided the original work is properly cited.

\section{Introduction}

Switching sources are devices used in the implementation of power converters. As a consequence of the switching action, chattering, high-order harmonic distortion, and nonlinear phenomena appear. The latter can be dealt with control techniques [1], while chattering and harmonic distortion, inherent to switching, can be reduced, but not avoided, using fixed switching frequency. To achieve this reduction, some techniques have been reported: adaptive hysteresis band [2,3], signal injection with a selected frequency [3-7], zero average current in each iteration (ZACE) [8], and recently zero average error dynamics in each iteration (ZAD) [9].

ZAD control scheme, proposed in [9], adds the advantages of fixed frequency implementations and the inherent robustness of sliding control modes. It is based on an appropriate design of the duty cycle in such a way that the sliding surface average in each 


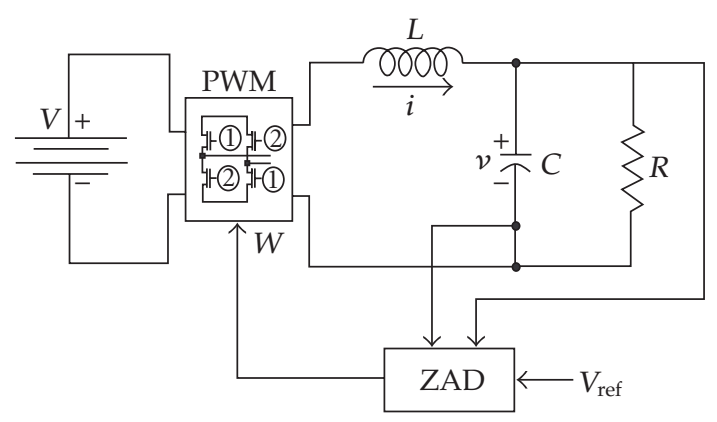

Figure 1: A schematic diagram of a buck power converter driven with PWM.

PWM period is zero and the output voltage tracks the reference with a very low error. A comparative study of this algorithm with regards to some other ones previously reported in the literature can be found in [10], while in [11], this ZAD technique was applied to a linear converter, showing good numeric and experimental results. In [12], a stability analysis and the chaos transition were explained and proved.

There are many studies in the averaging theory, mainly used for skipping the high frequency phenomenon. In fact, in many papers, the 1-periodic orbit is calculated invoking average theory. However, there are no studies devoted to bound the error in the system. The aim of this paper is to use the average theory $[13,14]$ for finding the maximum error of the system, taking into account the evolution of the trajectory in the continuous time, not only in the sampled time. This is important in the applications since usually only the sampled-time evolution is known. In our paper, we explicitly compute the error bounds along the whole continuous-time trajectory, giving a correct insight into the error dynamics. Then, centered and lateral ZAD-PWM schemes will be considered and steady-state maximum values for the error and the sliding surface in a sampling period will be computed.

At the end of the paper, an important lemma is proved. It relies on properties of transformations and stability of linear systems. With this lemma, it is possible to find the maximum error displayed for any ZAD-controlled stable second-order system with relative degree 2 and unitary gain, using the procedure shown in the paper.

The existence and stability of the 1-periodic orbit is assumed. This assumption is essential since the procedure is based on the 1-periodic orbit bound, then it cannot be applied to higher-period periodic orbits, as it can be checked with the results shown at the end of Section 4.

The paper is organized as follows: Section 2 is devoted to show the ZAD-PWM schemes for a buck converter and to give some generalities on average theory; bounds for the error and the sliding surface are computed in Section 3, for lateral and centered PWM; in Section 4 the obtained results are applied to a particular converter widely studied in the literature; in Section 5, these results are generalized, in order to be applied to any secondorder linear system; finally, conclusions are collected in the Section 6.

\section{Preliminaries}

Figure 1 depicts a simplified model of a buck power converter. 
This system can be modelled by the switched linear system

$$
\frac{d}{d r}\left(\begin{array}{c}
v \\
i
\end{array}\right)=\left(\begin{array}{cc}
-\frac{1}{R C} & \frac{1}{C} \\
-\frac{1}{L} & 0
\end{array}\right)\left(\begin{array}{l}
v \\
i
\end{array}\right)+\left(\begin{array}{c}
0 \\
\frac{V}{L}
\end{array}\right) W, \quad Y=v
$$

where the voltage in the capacitor $v(r)$ and the current in the inductor $i(r)$ are the state variables. The control signal $W$ takes discrete values in the set $\{-1,1\}$ depending on the switch position. The independent time variable is noted as $r$ and the sampling time as $T_{c}$. Let us define the dimensionless variables $z_{1}(\tau)=v / V, z_{2}(\tau)=(1 / V) \sqrt{(L / C)} i$ and $\tau=r / \sqrt{L C}$ and a new parameter $\gamma=(1 / R) \sqrt{L / C}$. The sampling time in the new variables is $\varepsilon=T_{c} / \sqrt{L C}$. The dimensionless dynamics can be written as

$$
\frac{d}{d \tau}\left(\begin{array}{l}
z_{1} \\
z_{2}
\end{array}\right)=\left(\begin{array}{ll}
-\gamma & 1 \\
-1 & 0
\end{array}\right)\left(\begin{array}{l}
z_{1} \\
z_{2}
\end{array}\right)+\left(\begin{array}{l}
0 \\
1
\end{array}\right) u, \quad y=z_{1}
$$

where $u \in\{-1,1\}$ is the input control which is implemented through a Pulse Width Modulator (PWM). The output of the system, corresponding to the controlled variable is $y=z_{1}$. This system will be controlled through a ZAD-PWM, guaranteeing Zero Average Dynamics (ZAD) for an auxiliary variable $s(\mathbf{z})$. This makes the output $z_{1}$ track a dimensionless reference voltage $v_{\text {ref }}=V_{\text {ref }} / V$. Since the output $y$ is relative degree 2 , and for robustness purposes, $s(\mathbf{z})$ is defined by [15]

$$
s(\mathbf{z})=\left(z_{1}-v_{\text {ref }}\right)+k_{s}\left(\dot{z}_{1}-\dot{v}_{\text {ref }}\right),
$$

where $z_{1}$ is the variable to be controlled and $k_{s}$ is the time-constant associated to the error dynamics. As the work of Carpita lies in the frame of sliding mode control, $s(\mathbf{z})$ is also named sliding surface. Strictly speaking, the discrete dynamics should be denoted as quasisliding, since the $s(\mathbf{z})$ samples lie on $s(\mathbf{z})>0$ and on $s(\mathbf{z})<0$, consecutively.

The ZAD-PWM strategy is based on computing a duty cycle, $D$. In this case, the duty cycle is the interval of time while the signal control is $u=1$. The ZAD-PWM strategy is obtaining $D$ such that

$$
\int_{k \varepsilon}^{(k+1) \varepsilon} s(\mathbf{z}(\tau)) d \tau=0
$$

where $\varepsilon$ is small and it is known as the dimensionless sampling (PWM) period. Then, the sliding surface $s(\mathbf{z})$ has zero average in each PWM period. The exact calculation of $D$ requires solving a transcendental equation. This is a problem for online implementations which is easily overcome approximating the original sliding surface by a piecewise linear one, which will be denoted also $s(\mathbf{z})$. For this reason, in [9-11, 16], a simplification was made and $s(\mathbf{z})$ was considered as piecewise straight line. However, no analytical results had shown the kindness of the technique. In [16], a first approximation to the proof of the advantages was done. In this paper, we use averaging techniques for proving that the approximation for $s(\mathbf{z})$ is good, and we find a bound for the maximum error in the system.

Let us consider $v_{\text {ref }}$ as a constant, and we rewrite the system dynamics in the more suitable variables: $e=z_{1}-v_{\text {ref }}$ (the output error) and $s=e+k_{s} \dot{e}$ (the sliding surface). In the following, we note this variables as $x_{1}$ and $x_{2}$, respectively. As we said previously, the period 
of the PWM is $\varepsilon$. Doing the change of variable $t=\tau / \varepsilon$, the duty cycle is now $d=D / \varepsilon$ and the equations of the system are

$$
\left(\begin{array}{c}
\dot{x}_{1} \\
\dot{x}_{2}
\end{array}\right)=\varepsilon\left(\begin{array}{cc}
-\frac{1}{k_{s}} & \frac{1}{k_{s}} \\
\gamma-\frac{1}{k_{s}}-k_{s} & \frac{1}{k_{s}}-\gamma
\end{array}\right)\left(\begin{array}{l}
x_{1} \\
x_{2}
\end{array}\right)+k_{s} \varepsilon\left(\begin{array}{c}
0 \\
u-v_{\text {ref }}
\end{array}\right), \quad y=x_{1}+v_{\text {ref }} .
$$

Now, the new independent time is $t$, and the period of the PWM is 1 . The system reads as $\dot{x}=\varepsilon f(x, t)$ as usual in averaging (perturbation) methods. The PWM provides the system with a width pulse, which is, roughly speaking, used to control the system. The pulse can be centered (CPWM) or lateral (LPWM), as it is illustrated in Figure 2. For the full-bridge buck converter and centered PMW, $u$ is defined as

$$
u= \begin{cases}1 & \text { if } k \leqslant t \leqslant k+\frac{d}{2} \\ -1 & \text { if } k+\frac{d}{2}<t<k+1-\frac{d}{2}, \\ 1 & \text { if } k+\left(1-\frac{d}{2}\right) \leqslant t \leqslant k+1,\end{cases}
$$

and for lateral pulse, width modulation $u$ is defined as

$$
u= \begin{cases}1 & \text { if } k \leqslant t \leqslant k+d \\ -1 & \text { if } k+d<t<k+1\end{cases}
$$

Equation (2.5) can be written in compact form as $\dot{\mathbf{x}}=\varepsilon \mathbf{A} \mathbf{x}+k_{s} \varepsilon \mathbb{U}$, where

$$
\mathbb{U}=\left(\begin{array}{c}
0 \\
u-v_{\text {ref }}
\end{array}\right), \quad \mathbf{A}=\left(\begin{array}{cc}
-\frac{1}{k_{s}} & \frac{1}{k_{s}} \\
\gamma-\frac{1}{k_{s}}-k_{s} & \frac{1}{k_{s}}-\gamma
\end{array}\right)
$$

being $u$ defined by (2.6) or (2.7), depending on the pulse generation scheme (CPWM or LPWM).

Remark 2.1. When the system operates in a 1-periodic orbit the following statements are fulfilled.

(i) Since $x_{2}=s$ independently of the scheme of the pulse (centered or lateral), the chosen control technique according to (2.4) implies $\left\langle x_{2}\right\rangle=0$ in each iteration. $\langle\cdot\rangle$ means average value, that is, $\int_{k}^{k+1} x_{2}(t) d t=0$.

(ii) As $\left\langle x_{2}\right\rangle=0$, then $\left\langle\dot{x}_{2}\right\rangle=0$.

(iii) The periodicity of $x_{1}$ implies $\left\langle\dot{x}_{1}\right\rangle=0$. With this consideration and $\left\langle x_{2}\right\rangle=0$ and taking into account the first equation of the (2.5), then $\left\langle x_{1}\right\rangle$ is forced to be zero $\left(\left\langle x_{1}\right\rangle=0\right)$.

(iv) Replacing these averaged values in the second equation of (2.5), it is possible to obtain $\left\langle u-v_{\text {ref }}\right\rangle=0$. 


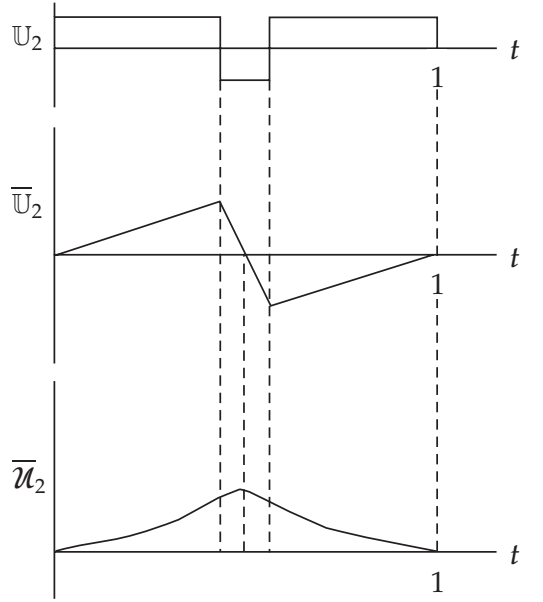

(a)

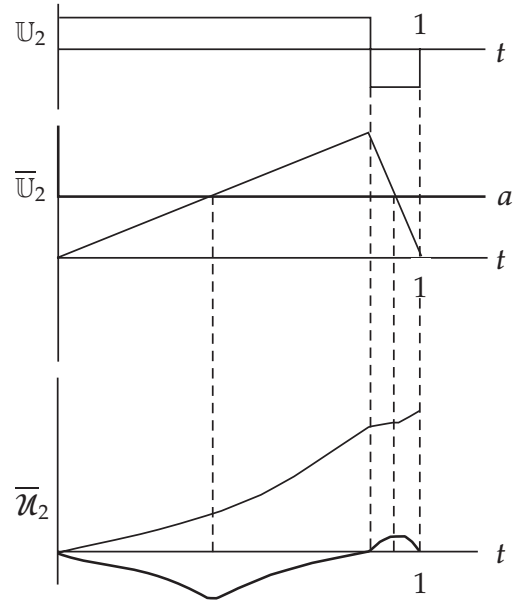

(b)

Figure 2: $\mathbb{U}_{2}$ and two first integrals. In (a) the CPWM case and in (b) the LPWM case.

Corollary 2.2. The duty cycle for the 1-periodic solution of system (2.5) is given by $d^{*}=0.5\left(1+v_{\text {ref }}\right)$.

Proof. It does not matter which pulse generation scheme (centered or lateral) we use, this value of $d$ guarantees $\langle\mathbb{U}\rangle=0$. This value is unique and it is obtained from the solution of the following equations, depending on the generation pulse scheme:

$$
\int_{0}^{d / 2}\left(1-v_{\text {ref }}\right) d t+\int_{d / 2}^{1-d / 2}\left(-1-v_{\text {ref }}\right) d t+\int_{1-d / 2}^{1}\left(1-v_{\text {ref }}\right) d t=0
$$

or

$$
\int_{0}^{d}\left(1-v_{\text {ref }}\right) d t+\int_{d}^{1}\left(-1-v_{\text {ref }}\right) d t=0
$$

Remark 2.3. Before we define some changes of variables, let us consider a generic system $\dot{x}=\varepsilon A x+\varepsilon \mathbb{U}$, where $\mathbb{U}$ has jump discontinuities. First, assume that $\mathbb{U}$ is $\mathcal{C}^{\infty}$ in $[0,1]$, except at a finite number of points $p_{i} \in[0,1]$. Second, let $x(t)$ be a solution of the differential equation $\dot{x}=\varepsilon A x+\varepsilon \mathbb{U}$. It is continuous in all $[0,1]$ and $\mathcal{C}^{\infty}$ in $[0,1]$ except at the points $p_{i}$. Third, if we define $\bar{u}=\int_{0}^{t} \mathbb{U}$, this is also $\mathcal{C}^{\infty}$ in all $[0,1]$ except at the points $p_{i}$, where it is only continuous. Let us define $y=x-\varepsilon \bar{u}, y$ is $\mathcal{C}^{\infty}$ everywhere except at the points $p_{i}$, where, in principle, it is continuous. This change of variables can be done in each subinterval $\left[p_{i}, p_{i+1}\right]$ and extended everywhere due to the continuity of $\bar{u}$ at $p_{i}$. Finally, one obtains

$$
\dot{y}=\varepsilon A y+\varepsilon^{2} A \bar{u}
$$

The function $f(t, y)=\varepsilon A y+\varepsilon^{2} \bar{u}$ is continuous with regards to $t$ and $\mathcal{C}^{1}$ with regards to $y$. The ODE existence theorem states that all the solutions of equation $\dot{y}=\varepsilon A y+\varepsilon^{2} A \bar{u}$ are $C^{1}$. This allows us to do the proposed change of variables, which is common in averaging theory. 
Subsequently, in order to average the system, let us define the change of variables $\mathbf{y}=\mathbf{x}-\varepsilon \overline{\mathbb{U}}$, where

$$
\overline{\mathbb{U}}=k_{s} \int_{0}^{t} \mathbb{U} d \tau
$$

Since $\langle\mathbb{U}\rangle=0$, then $\overline{\mathbb{U}}$ is periodic. It is straightforward to obtain

$$
\dot{\mathbf{y}}=\varepsilon \mathbf{A y}+\varepsilon^{2} \mathbf{A} \overline{\mathbb{U}}
$$

For the next change of variables, let us note that we cannot assume that the function $\int_{0}^{t} \mathbf{A} \overline{\mathbb{U}}$ is periodic. The behavior for the CPWM and LPWM cases are shown in Figure 2. To solve this problem, let us define the mean of $\mathbf{A} \overline{\mathbb{U}}$ as

$$
\begin{gathered}
\mathbf{a}=\int_{0}^{1} \mathbf{A} \overline{\mathbb{U}} d t, \\
\overline{\mathcal{U}}=\int_{0}^{t}(\mathbf{A} \overline{\mathbb{U}}-\mathbf{a}) d \tau .
\end{gathered}
$$

Note that $\overline{\mathcal{U}}$ is periodic. Now, let us define a new change of variables, namely,

$$
\mathbf{z}=\mathbf{y}-\varepsilon^{2} \bar{u}
$$

Then,

$$
\dot{\mathbf{z}}=\dot{\mathbf{y}}-\varepsilon^{2} \dot{\bar{u}}
$$

holds.

This equation is also well defined in the switching instant because $\bar{u}$ is $\mathcal{C}^{1}$. Hence, the following equation holds

$$
\dot{\mathbf{z}}=\dot{\mathbf{y}}-\varepsilon^{2}(\mathbf{A} \overline{\mathbb{U}}-\mathbf{a}) .
$$

Replacing (2.13) and (2.16) in (2.18) yields to

$$
\dot{\mathbf{z}}=\varepsilon \mathbf{A z}+\varepsilon^{2} \mathbf{a}+\varepsilon^{3} \mathbf{A} \overline{\mathcal{u}}
$$

Assuming $\bar{u}=\mathbf{0}$, we find the equilibrium point of equation (2.19) as $\mathbf{z}^{*}=-\varepsilon \mathbf{A}^{-1} \mathbf{a}$. The invertibility of the matrix $\mathbf{A}$ is assured by the eigenvalues of the systems (they are different from zero). Then, (2.19) can be expressed as

$$
\dot{\mathbf{w}}=\varepsilon \mathbf{A} \mathbf{w}+\varepsilon^{3} \mathcal{W}
$$

where $\mathbf{w}=\mathbf{z}-\mathbf{z}^{*}$ and $\mathcal{W}=\mathbf{A} \overline{\mathcal{U}}$. The general solution for this equation is

$$
\mathbf{w}(t)=e^{\varepsilon \mathbf{A} t} \mathbf{w}(0)+\varepsilon^{3} \int_{0}^{t} e^{\varepsilon \mathbf{A}(t-\sigma)} \mathcal{W}(\sigma) d \sigma .
$$


A steady-state 1-periodic solution satisfies $\mathbf{w}(1)=\mathbf{w}(0)$. Hence,

$$
\mathbf{w}(0)=\left(\mathbf{I}-e^{\varepsilon \mathbf{A}}\right)^{-1} \varepsilon^{3} \int_{0}^{1} e^{\varepsilon \mathbf{A}(1-\sigma)} \mathcal{W}(\sigma) d \sigma .
$$

Finally, the general solution in the original variables $\mathbf{x}$ of the system is

$$
\mathbf{x}(t)=\varepsilon \overline{\mathbb{U}}(t)+\varepsilon^{2} \overline{\mathcal{U}}(t)+\mathbf{w}(t)-\varepsilon \mathbf{A}^{-1} \mathbf{a},
$$

where $\mathbf{w}(t)$ is given by (2.21) and (2.22). Then, for bounding $x_{1}(t)(e(t))$ and $x_{2}(t)(s(t))$, it is necessary to bound all terms of (2.23). The terms $\overline{\mathbb{U}}$ and $\overline{\mathcal{U}}$ depend only on the input control signal; and their integrals are well known. The term $\mathbf{A}^{-1}$ a depends on the parameters of the system and on the input signal. For this reason, these three terms are easily bounded. Then, in order to bound $\mathbf{x}(t)$ (defined by (2.23)), we will proceed by obtaining bounds for each component of the variables $\mathbf{w}(t)$ and $\mathbf{w}(0)$. This implies to bound $e^{\varepsilon \mathbf{A} t},\left(\mathbf{I}-e^{\varepsilon \mathbf{A}}\right)^{-1}$ and $\int_{0}^{1} e^{\varepsilon \mathbf{A}(1-\sigma)} \mathcal{W}(\sigma) d \sigma$.

\section{Bounds}

This section is devoted to obtain bounds of the 1-periodic solution in terms of the input control signal and the constant matrix.

Since the expression for $\mathcal{W}$ differs depending on the lateral or centered pulses, the analysis is done separately. However, the common terms admit a unique analysis. In addition, since the differences in magnitude between variables $x_{1}$ and $x_{2}$ have to be taken into account, it is better to analyze each one separately. This will be done in the following subsections.

\subsection{State transition matrix}

The state transition matrix can be written in compact form as

$$
e^{\varepsilon \mathbf{A} t}=\left(\begin{array}{ll}
e_{11}(t) & e_{12}(t) \\
e_{21}(t) & e_{22}(t)
\end{array}\right),
$$

where

$$
\begin{gathered}
e_{11}(t)=e^{(-\gamma / 2) \varepsilon t}\left(\frac{\gamma / 2-1 / k_{s}}{\alpha} \sin (\alpha \varepsilon t)+\cos (\alpha \varepsilon t)\right), \quad e_{12}(t)=e^{(-\gamma / 2) \varepsilon t} \frac{1}{\alpha k_{s}} \sin (\alpha \varepsilon t), \\
e_{21}(t)=e^{(-\gamma / 2) \varepsilon t} \frac{\left(\gamma-k_{s}-1 / k_{s}\right)}{\alpha} \sin (\alpha \varepsilon t), \quad e_{22}(t)=e^{(-\gamma / 2) \varepsilon t}\left(\frac{-\gamma / 2+1 / k_{s}}{\alpha} \sin (\alpha \varepsilon t)+\cos (\alpha \varepsilon t)\right),
\end{gathered}
$$

and $\alpha=\sqrt{1-\gamma^{2} / 4}$. Expanding these coefficients in a Taylor series up to first order and evaluating its maximum in the interval $\left[\begin{array}{ll}0 & 1\end{array}\right]$ yields to

$$
\begin{gathered}
\max \left|e_{11}(t)\right| \leqslant\left|\frac{\gamma}{2}-\frac{1}{k_{s}}\right| \varepsilon+1:=\left|e_{11}\right|, \quad \max \left|e_{12}(t)\right| \leqslant \frac{1}{k_{s}} \varepsilon:=\left|e_{12}\right|, \\
\max \left|e_{21}(t)\right| \leqslant\left|\gamma-k_{s}-\frac{1}{k_{s}}\right| \varepsilon:=\left|e_{21}\right|, \quad \max \left|e_{22}(t)\right| \leqslant\left|\frac{\gamma}{2}-\frac{1}{k_{s}}\right| \varepsilon+1:=\left|e_{22}\right| .
\end{gathered}
$$




\subsection{Inverse of $\left(\mathbf{I}-e^{\varepsilon \mathrm{A}}\right)$}

In this section, and in order to get accurate bounds, we expand all the functions by Taylor up to second order.

$\left(\mathbf{I}-e^{\varepsilon \mathbf{A} t}\right)^{-1}$ can be expressed as

$$
\left(\mathbf{I}-e^{\varepsilon \mathbf{A} t}\right)^{-1}=\frac{1}{\operatorname{det}\left(\mathbf{I}-e^{\varepsilon \mathbf{A} t}\right)}\left(\begin{array}{ll}
m_{11}(t) & m_{12}(t) \\
m_{21}(t) & m_{22}(t)
\end{array}\right),
$$

where $m_{11}(t)=1-e_{22}(t), m_{12}(t)=e_{12}(t), m_{21}(t)=e_{21}(t), m_{22}(t)=1-e_{11}(t)$, and $\operatorname{det}\left(\mathbf{I}-e^{\varepsilon \mathbf{A} t}\right)=$ $1-2 e^{(-\gamma / 2) \varepsilon t} \cos (\alpha \varepsilon t)+e^{-\gamma \varepsilon t}$. Since order $1(O(1))$ and order $\varepsilon t(O(\varepsilon t))$ terms are cancelled, second-order Taylor series expansion is required. Hence,

$$
\begin{gathered}
\cos (\alpha \varepsilon t)=1-\frac{1}{2}(\alpha \varepsilon t)^{2}+O\left((\alpha \varepsilon t)^{3}\right)=1-\frac{1}{2}(\alpha \varepsilon t)^{2}+\epsilon_{1}, \\
e^{(-\gamma / 2) \varepsilon t}=1-\frac{\gamma \varepsilon t}{2}+\frac{1}{2}\left(\frac{\gamma \varepsilon t}{2}\right)^{2}+O\left(\left(\frac{\gamma \varepsilon t}{2}\right)^{3}\right)=1-\frac{\gamma \varepsilon t}{2}+\frac{1}{2}\left(\frac{\gamma \varepsilon t}{2}\right)^{2}+\epsilon_{2}, \\
e^{-\gamma \varepsilon t}=1-\gamma \varepsilon t+\frac{1}{2}(\gamma \varepsilon t)^{2}+O\left((\gamma \varepsilon t)^{3}\right)=1-\gamma \varepsilon t+\frac{1}{2}(\gamma \varepsilon t)^{2}+\epsilon_{3},
\end{gathered}
$$

where $\epsilon_{1}, \epsilon_{2}$, and $\epsilon_{3}$ correspond to higher-order error terms. From Taylor theorem, $\left|\epsilon_{1}\right| \leqslant$ $(\alpha \epsilon t)^{3} / 6,\left|\epsilon_{2}\right| \leqslant(1 / 6)(\gamma \epsilon t / 2)^{3}$, and $\left|\epsilon_{3}\right| \leqslant(\gamma \epsilon t)^{3} / 6$, and the determinant is reduced to

$$
\operatorname{det}\left(\mathbf{I}-e^{\varepsilon \mathbf{A} t}\right)=\left(\varepsilon^{2} t^{2}+E\right),
$$

where $E=-2 e^{(-\gamma / 2) \varepsilon t} \epsilon_{1}-2 \cos (\alpha \varepsilon t) \epsilon_{2}+\epsilon_{3}+(1 / 8) \gamma^{2} \alpha^{2} \varepsilon^{4} t^{4}-(1 / 2) \gamma \alpha^{2} \varepsilon^{3} t^{3}$.

The leading terms of the adjoint matrix coefficients are of order $O(\varepsilon t)$. Hence, in the Taylor series expansion, they must be developed until order 1 . The following expansions are used:

$$
\begin{gathered}
\cos (\alpha \varepsilon t)=1+O\left((\alpha \varepsilon t)^{2}\right)=1+\epsilon_{4}, \\
\sin (\alpha \varepsilon t)=\alpha \varepsilon t+O\left((\alpha \varepsilon t)^{2}\right)=\alpha \varepsilon t+\epsilon_{5}, \\
e^{(-\gamma / 2) \varepsilon t}=1-\frac{\gamma \varepsilon t}{2}+O\left(\left(\frac{\gamma \varepsilon t}{2}\right)^{2}\right)=1-\frac{\gamma \varepsilon t}{2}+\epsilon_{6},
\end{gathered}
$$

where $\epsilon_{4} \leqslant(\alpha \varepsilon t)^{2} / 2, \epsilon_{5} \leqslant(\alpha \varepsilon t)^{2} / 2$, and $\epsilon_{6} \leqslant(1 / 2)(\gamma \varepsilon t / 2)^{2}$. Also, the coefficients $m_{i j}$ can be written as

$$
\begin{gathered}
m_{11}=\left(r-\frac{1}{k_{s}}\right) t \varepsilon+\epsilon_{m 11}, \quad m_{12}=\frac{1}{k_{s}} t \varepsilon+\epsilon_{m 12}, \\
m_{21}=\left(r-\frac{1}{k_{s}}-k_{s}\right) t \varepsilon+\epsilon_{m 21}, \quad m_{22}=\frac{1}{k_{s}} t \varepsilon+\epsilon_{m 22},
\end{gathered}
$$

where

$$
\begin{gathered}
\epsilon_{m 11}=-c_{1} e^{(-\gamma / 2) \varepsilon t} \epsilon_{5}-c_{1} \sin (\alpha \varepsilon t) \epsilon_{6}-e^{(-\gamma / 2) \varepsilon t} \epsilon_{4}-\cos (\alpha \varepsilon t) \epsilon_{6}+c_{1} \frac{\alpha \gamma}{2} \varepsilon^{2} t^{2}, \\
\epsilon_{m 12}=-\frac{1}{\alpha k_{s}} e^{(-\gamma / 2) \varepsilon t} \epsilon_{5}-\frac{1}{\alpha k_{s}} \sin (\alpha \varepsilon t) \epsilon_{6}+\frac{\gamma}{2 k_{s}} \varepsilon^{2} t^{2}, \\
\epsilon_{m 21}=c_{2} e^{(-\gamma / 2) \varepsilon t} \epsilon_{5}+c_{2} \sin (\alpha \varepsilon t) \epsilon_{6}+c_{2} \frac{\alpha \gamma}{2} \varepsilon^{2} t^{2}, \\
\epsilon_{m 22}=c_{1} e^{(-\gamma / 2) \varepsilon t} \epsilon_{5}+c_{1} \sin (\alpha \varepsilon t) \epsilon_{6}-e^{(-\gamma / 2) \varepsilon t} \epsilon_{4}-\cos (\alpha \varepsilon t) \epsilon_{6}-c_{1} \frac{\alpha \gamma}{2} \varepsilon^{2} t^{2},
\end{gathered}
$$


where $c_{1}=1 / \alpha k_{s}-\gamma / 2 \alpha$ and $c_{2}=\left(k_{s}+1 / k_{s}-\gamma\right) / \alpha$. Finally, let us take $t=1$ and bound the errors as an addition of the absolute values of the addends. Exponential and cosine functions are bounded by 1 , and sine function is bounded by the angle; hence, the expressions for each error term are

$$
\begin{gathered}
|E| \leqslant 2\left|\frac{\alpha^{3} \varepsilon^{3}}{6}\right|+\frac{1}{24}\left|\gamma^{3} \varepsilon^{3}\right|+\frac{1}{6}\left|\gamma^{3} \varepsilon^{3}\right|+\left|\left(\frac{1}{8} \gamma^{2} \alpha^{2} \varepsilon-\frac{1}{2} \gamma \alpha^{2}\right) \varepsilon^{3}\right|:=\varepsilon^{3}\left|E^{*}\right|, \\
\left|\epsilon_{m 11}\right| \leqslant\left|c_{1} \frac{\alpha^{2} \varepsilon^{2}}{2}\right|+\left|c_{1} \alpha \frac{r^{2} \varepsilon^{3}}{8}\right|+\left|\frac{\alpha^{2} \varepsilon^{2}}{2}\right|+\left|\frac{r^{2} \varepsilon^{2}}{8}\right|+\left|c_{1} \alpha \frac{\gamma}{2} \varepsilon^{2}\right|, \\
\left|\epsilon_{m 12}\right| \leqslant\left|\frac{1}{2 k_{s}} \varepsilon^{2}\right|+\left|\frac{\gamma^{2}}{8 k_{s}} \varepsilon^{3}\right|+\left|\frac{\gamma}{2 k_{s}} \varepsilon^{2}\right|, \\
\left|\epsilon_{m 21}\right| \leqslant\left|c_{2} \frac{\alpha^{2}}{2} \varepsilon^{2}\right|+\left|c_{2} \alpha \frac{r^{2}}{8} \varepsilon^{3}\right|+\left|c_{2} \alpha \frac{\gamma}{2} \varepsilon^{2}\right|, \\
\left|\epsilon_{m 22}\right| \leqslant\left|c_{1} \frac{\alpha^{2} \varepsilon^{2}}{2}\right|+\left|c_{1} \alpha \frac{r^{2} \varepsilon^{3}}{8}\right|+\left|\frac{\alpha^{2} \varepsilon^{2}}{2}\right|+\left|\frac{\gamma^{2} \varepsilon^{2}}{8}\right|+\left|c_{1} \alpha \frac{\gamma}{2} \varepsilon^{2}\right|,
\end{gathered}
$$

where $\left|E^{*}\right| \leqslant \alpha^{3} / 3+(5 / 24) \gamma^{3}+\left(\alpha^{2} \gamma / 2\right)|((\gamma / 4) \varepsilon-1)|$. Then, assuming $\varepsilon\left|E^{*}\right|<1$ and $t=1$, we obtain a lower bound for each term as

$$
\begin{gathered}
\left|\operatorname{det}\left(\mathbb{I}-e^{\varepsilon \mathbf{A}}\right)\right| \geqslant \Delta=\varepsilon^{2}\left(1-\varepsilon\left|E^{*}\right|\right), \\
\left|m_{11}\right| \leqslant\left|\gamma-\frac{1}{k_{s}}\right| \varepsilon+\left|\epsilon_{m 11}\right|, \quad\left|m_{12}\right| \leqslant\left|\frac{1}{k_{s}}\right| \varepsilon+\left|\epsilon_{m 12}\right|, \\
\left|m_{21}\right| \leqslant\left|\gamma-k_{s}-\frac{1}{k_{s}}\right| \varepsilon+\left|\epsilon_{m 21}\right|, \quad\left|m_{22}\right| \leqslant\left|\frac{1}{k_{s}}\right| \varepsilon+\left|\epsilon_{m 22}\right| .
\end{gathered}
$$

The aim of the following sections is to evaluate $\mathbf{w}(0)$ in $(2.22)$ and $\mathbf{w}(t)$ in $(2.21)$ in order to obtain the maximum of the error $x_{1}(t)$ and of the sliding surface $x_{2}(t)$ of (2.23). As (2.21) and (2.22) depend on the pulse generation scheme (centered pulse or lateral pulse), the analysis are done independently.

\section{3. $\mathrm{w}(0)$ and $\mathrm{w}(t)$ for centered pulse width modulator (CPWM)}

Taking into account that $\mathbf{w}(t)$ is given by

$$
\mathbf{w}(t)=e^{\varepsilon \mathbf{A} t} \mathbf{w}(0)+\varepsilon^{3} \int_{0}^{t} e^{\varepsilon \mathbf{A}(t-\sigma)} \mathcal{W}(\sigma) d \sigma
$$

where

$$
\mathbf{w}(0)=\left(\mathbf{I}-e^{\varepsilon \mathbf{A}}\right)^{-1} \varepsilon^{3} \int_{0}^{1} e^{\varepsilon \mathbf{A}(1-\sigma)} \mathcal{W}(\sigma) d \sigma,
$$


in order to establish the maximum value of $\mathbf{w}(t)$, we need to compute the maximum values taken for each component of vector $\mathbf{w}(0)$. For doing this, first we compute $\mathcal{W}$. From (2.5) and (2.6), we have

$$
\begin{gathered}
\mathbb{U}=\left(\begin{array}{c}
0 \\
u-v_{\text {ref }}
\end{array}\right):=\left(\begin{array}{l}
\mathbb{U}_{1} \\
\mathbb{U}_{2}
\end{array}\right), \\
\mathbb{U}_{2}= \begin{cases}1-v_{\text {ref }} & \text { if } 0 \leqslant t \leqslant \frac{d}{2}, \\
-1-v_{\text {ref }} & \text { if } \frac{d}{2}<t \leqslant 1-\frac{d}{2}, \\
1-v_{\text {ref }} & \text { if } 1-\frac{d}{2}<t \leqslant 1,\end{cases}
\end{gathered}
$$

$d$ is the time the switch takes value +1 . As we said previously, the duty cycle in the steady state is given by $d=0.5\left(1+v_{\text {ref }}\right)$. From the definition of $\overline{\mathbb{U}}$, we have

$$
\overline{\mathbb{U}}=k_{s} \int_{0}^{t} \mathbb{U} d \tau:=\left(\overline{\mathbb{U}}_{1} \overline{\mathbb{U}}_{2}\right)^{T}
$$

where $\overline{\mathbb{U}}_{1}=0$ and $\overline{\mathbb{U}}_{2}$ are periodic signals given by

$$
\overline{\mathbb{U}}_{2}= \begin{cases}k_{s}\left(1-v_{\text {ref }}\right) t & \text { if } 0 \leqslant t \leqslant \frac{d}{2} \\ k_{s}\left(d-\left(1+v_{\text {ref }}\right) t\right) & \text { if } \frac{d}{2}<t \leqslant 1-\frac{d}{2} \\ k_{s}\left(2 d-2+\left(1-v_{\text {ref }}\right) t\right) & \text { if } 1-\frac{d}{2}<t<1 .\end{cases}
$$

From (2.14) and using (3.17), we have $\mathbf{a}=\mathbf{A} \int_{0}^{1} \overline{\mathbb{U}} d t=0$. Then, $\langle\overline{\mathbb{U}}\rangle=0$ and $\bar{u}=\int_{0}^{t}(\mathbf{A} \overline{\mathbb{U}}-\mathbf{a}) d \tau=$ $k_{s} \mathbf{A}\left(\begin{array}{ll}b_{1} & b_{2}\end{array}\right)^{T}$, where $b_{1}=0$ and

$$
b_{2}= \begin{cases}\frac{1}{2}\left(1-v_{\text {ref }}\right) t^{2} & \text { if } 0 \leqslant t \leqslant \frac{d}{2} \\ -\frac{1}{4} d^{2}+d t-\frac{1}{2}\left(1+v_{\text {ref }}\right) t^{2} & \text { if } \frac{d}{2}<t \leqslant 1-\frac{d}{2} \\ (1-d)-2(1-d) t+\frac{1}{2}\left(1-v_{\text {ref }}\right) t^{2} & \text { if } 1-\frac{d}{2}<t \leqslant 1\end{cases}
$$

which is also a periodic signal. Finally, $\overline{\mathfrak{U}}$ can be expressed in compact form as

$$
\bar{u}=\left(\begin{array}{c}
b_{2} \\
\left(1-k_{s} \gamma\right) b_{2}
\end{array}\right):=\left(\begin{array}{l}
\mathcal{U}_{1} \\
\mathcal{U}_{2}
\end{array}\right), \quad \mathcal{W}=\mathrm{A} \overline{\mathcal{U}}=\left(\begin{array}{c}
-\gamma b_{2} \\
\left(k_{s} \gamma^{2}-\gamma-k_{s}\right) b_{2}
\end{array}\right):=\left(\begin{array}{l}
\mathfrak{W}_{1} \\
\mathcal{W}_{2}
\end{array}\right) .
$$

Let us define

$$
\mathbf{I}=\int_{0}^{1} e^{\varepsilon \mathbf{A}(1-\sigma)} \mathcal{W}(\sigma) d \sigma
$$


Fabiola Angulo et al.

whose components $I_{i}$ fulfill

$$
\left|I_{i}\right| \leqslant\left(\gamma\left|e_{i 1}\right|+\left|k_{s} \gamma^{2}-k_{s}-\gamma\right|\left|e_{i 2}\right|\right)\left|\int_{0}^{1} b_{2}(\sigma) d \sigma\right| .
$$

By integrating the input $b_{2}(\sigma)$ on the interval $[0,1]$, we have

$$
\left|I_{i}\right| \leqslant\left(\gamma\left|e_{i 1}\right|+\left|k_{s} \gamma^{2}-k_{s}-\gamma\right|\left|e_{i 2}\right|\right)\left|\frac{1}{12}\left(1+v_{\text {ref }}\right)-\frac{1}{16}\left(1+v_{\text {ref }}\right)^{2}+\frac{1}{96}\left(1+v_{\text {ref }}\right)^{3}\right| .
$$

For the coefficients $\left|m_{i j}(t)\right|$ defined in (3.4), let $\left|m_{i j}\right|=\max \left|m_{i j}(t)\right|$ for all $0 \leqslant t \leqslant 1$. Then, (2.22) and (2.21) yield to

$$
\left|w_{i}(0)\right| \leqslant \varepsilon \frac{1}{|1-\varepsilon| E^{*}||}\left(\left|m_{i 1} I_{1}\right|+\left|m_{i 2} I_{2}\right|\right), \quad\left|w_{i}(t)\right| \leqslant\left|e_{i 1} w_{1}(0)\right|+\left|e_{i 2} w_{2}(0)\right|+\varepsilon^{3}\left|I_{i}\right| .
$$

Finally, using the estimated bound of $\mathbf{w}(t)$, it is easy to find the maximum values for the error $e=x_{1}$ and for the sliding surface $s=x_{2}$, using the following equation:

$$
\mathbf{x}(t)=\varepsilon \overline{\mathbb{U}}(t)+\varepsilon^{2} \overline{\mathcal{U}}(t)+\mathbf{w}(t) .
$$

\section{4. $\mathrm{w}(0)$ and $\mathrm{w}(t)$ for lateral pulse (LPWM)}

In this case, the pulse generation order is $+1-1$. As it was stated before, $d$ is the time the switch is +1 and it is given by $d=0.5\left(1+v_{\text {ref }}\right)$. Proceeding as in the previous subsection, let us define $\mathbb{U}=\left[\begin{array}{ll}\mathbb{U}_{1} & \mathbb{U}_{2}\end{array}\right]^{T}$, where $\mathbb{U}_{1}=0$ and

$$
\mathbb{U}_{2}= \begin{cases}1-v_{\text {ref }} & \text { if } 0 \leqslant t \leqslant d, \\ -1-v_{\text {ref }} & \text { if } d<t<1\end{cases}
$$

the evaluation of

$$
\overline{\mathbb{U}}=k_{s} \int_{0}^{t} \mathbb{U} d \tau:=\left[\overline{\mathbb{U}}_{1} \overline{\mathbb{U}}_{2}\right]^{T}
$$

yields to $\overline{\mathbb{U}}_{1}=0$ and

$$
\overline{\mathbb{U}}_{2}= \begin{cases}k_{s}\left(1-v_{\text {ref }}\right) t & \text { if } 0 \leqslant t \leqslant d, \\ k_{s}\left(2 d-\left(1+v_{\text {ref }}\right) t\right) & \text { if } d<t<1 .\end{cases}
$$

For this case, $\langle\overline{\mathbb{U}}\rangle \neq 0$. In order to evaluate the mean of $\overline{\mathbb{U}}$, let us compute

$$
\mathbf{a}=\mathbf{A} \int_{0}^{1} \overline{\mathbb{U}} d t=k_{s} \mathbf{A}\left(\begin{array}{c}
0 \\
-d^{2}+2 d-\frac{1}{2}-\frac{v_{\text {ref }}}{2}
\end{array}\right)=k_{s} \mathbf{A}\left(\begin{array}{l}
0 \\
\beta
\end{array}\right)
$$

being $\beta=-d^{2}+2 d-1 / 2-v_{\text {ref }} / 2$. 
However, $\overline{\mathcal{U}}=\int_{0}^{t}(\mathbf{A} \mathbb{U}(\sigma)-\mathbf{a}) d \sigma$ can be evaluated as

$$
\bar{u}=k_{s} \mathbf{A}\left(\begin{array}{ll}
b_{1} & b_{2}
\end{array}\right)^{T},
$$

where $b_{1}=0$ and

$$
b_{2}= \begin{cases}\frac{1}{2}\left(1-v_{\text {ref }}\right) t^{2}-\beta t & \text { if } 0 \leqslant t \leqslant d, \\ -d^{2}+(2 d-\beta) t-\frac{1}{2}\left(1+v_{\text {ref }}\right) t^{2} & \text { if } d<t \leqslant 1 .\end{cases}
$$

Finally, $\overline{\mathcal{U}}$ is given in compact form as

$$
\overline{\mathcal{U}}=\left(\begin{array}{c}
b_{2} \\
\left(1-k_{s} \gamma\right) b_{2}
\end{array}\right):=\left(\begin{array}{l}
\overline{\mathcal{U}}_{1} \\
\overline{\mathcal{U}}_{2}
\end{array}\right), \quad \boldsymbol{W}=\mathrm{A} \mathcal{W}=\left(\begin{array}{c}
-\gamma b_{2} \\
\left(k_{s} \gamma^{2}-\gamma-k_{s}\right) b_{2}
\end{array}\right):=\left(\begin{array}{l}
\mathcal{W}_{1} \\
\mathcal{W}_{2}
\end{array}\right) .
$$

As in the preceding case, the components of I function

$$
\mathbf{I}=\int_{0}^{1} e^{\varepsilon \mathbf{A}(1-\sigma)} \mathcal{W}(\sigma) d \sigma
$$

can be bounded by

$$
\left|I_{i}\right| \leqslant\left(\gamma\left|e_{i 1}\right|+\left|k_{s} \gamma^{2}-k_{s}-\gamma\right|\left|e_{i 2}\right|\right)\left|\int_{0}^{1} b_{2}(\sigma) d \sigma\right| .
$$

Integrating the input $b_{2}(\sigma)$ on the interval $[0,1]$ yields to

$$
\left|I_{i}\right| \leqslant\left(\gamma\left|e_{i 1}\right|+\left|k_{s} \gamma^{2}-k_{s}-\gamma\right|\left|e_{i 2}\right|\right)\left|\frac{1}{12}\left(1+v_{\text {ref }}\right)-\frac{1}{8}\left(1+v_{\text {ref }}\right)^{2}+\frac{1}{24}\left(1+v_{\text {ref }}\right)^{3}\right| .
$$

Finally,

$$
\left|w_{i}(0)\right| \leqslant \varepsilon \frac{1}{|1-\varepsilon| E^{*}||}\left(\left|m_{i 1} I_{1}\right|+\left|m_{i 2} I_{2}\right|\right), \quad\left|w_{i}(t)\right| \leqslant\left(\left|e_{i 1} w_{1}(0)\right|+\left|e_{i 2} w_{2}(0)\right|\right)+\varepsilon^{3}\left|I_{i}\right| .
$$

Using this bound for $\mathbf{w}(t)$, we find the bound for $e(t)$ and $s(t)$ using

$$
\mathbf{x}(t)=\varepsilon \overline{\mathbb{U}}(t)+\varepsilon^{2} \overline{\mathcal{U}}(t)+\mathbf{w}(t)-\varepsilon \mathbf{A}^{-1} \mathbf{a},
$$

where $e=x_{1}$ and $s=x_{2}$. It is worth to note that in both cases, the output error is defined by

$$
e(t)=w_{1}(t)+\varepsilon^{2} \bar{u}_{1}^{2}
$$

which implies a very low error. 


\section{Estimation of the maximum values for the output error and the sliding surface}

Let us now compute all of these calculus for the parameters values used in $[11,12,16,17]$. These parameters are $\gamma=0.35, v_{\text {ref }}=0.8$, and $\varepsilon=T=0.1767$, and they correspond to $R=$ $20 \Omega, C=40 \mu \mathrm{F}, L=2 \mathrm{mH}, V_{\text {ref }}=32 \mathrm{~V}, V=40 \mathrm{~V}$, and $T_{c}=50 \mu \mathrm{s}$ in a physical system. With the value of $v_{\text {ref }}$, we obtain $d=0.9$.

\subsection{CPWM}

In [12], it was proved that the limit of the stability is close to $k_{s}=3.24$. Values below this point lead the system to other operation point. Then, for obtaining a 1-periodic orbit, we use $k_{s}=4.5$. The following bounds were found $\operatorname{det}\left(I-e^{\varepsilon \mathbf{A}}\right) \geqslant 0.0285,\left|e_{11}\right| \leqslant 1.0083,\left|e_{12}\right| \leqslant$ $0.0393,\left|e_{21}\right| \leqslant 0.7726,\left|e_{22}\right| \leqslant 1.0083, I_{1} \leqslant 0.0043, I_{2} \leqslant 0.0379,\left|m_{11}\right| \leqslant 0.0392,\left|m_{12}\right| \leqslant$ $0.0440,\left|m_{21}\right| \leqslant 0.8640$, and $\left|m_{22}\right| \leqslant 0.0559$. Hence, the following hold

$$
\begin{array}{ll}
w_{1}(0) \leqslant 0.00036, & w_{2}(0) \leqslant 0.0011 \\
w_{1}(t) \leqslant 0.00043, & w_{2}(t) \leqslant 0.0016 .
\end{array}
$$

\subsubsection{Output error estimation}

Using (3.24) and taking into account that the first component of the input vector $\mathbb{U}$ is zero, error dynamics is defined by the first components of $\mathbf{w}(t)$ and $\varepsilon^{2} \overline{\mathcal{U}}$, thus,

$$
e(t)=w_{1}(t)+\varepsilon^{2} \bar{u}_{1}
$$

The maximum of $\left|\overline{\mathfrak{U}}_{1}\right|=\left|b_{2}\right|$ holds at $t=0.5$ and it is 0.0225 , then

$$
\max |e(t)| \leqslant 0.0011
$$

This is equivalent to a maximum error value of $0.14 \%$ in steady state when the reference reaches the value 0.8 . The error behavior obtained from a numerical simulation is depicted in Figure 3. Note that the real error value is lower than the estimated one. However, the bound is really close.

\subsubsection{Sliding surface error estimation}

In this case, the expression is adjusted to analyze the second component as

$$
s(t)=\varepsilon \overline{\mathbb{U}}_{2}+\varepsilon^{2} \overline{\mathfrak{U}}_{2}+w_{2}(t)
$$

The maximum associated to $\left|\overline{\mathbb{U}}_{2}\right|$ holds at $t=0.45$ and it is 0.4050 ; while the maximum of $\left|\overline{\mathcal{U}}_{2}\right|=\left|\left(1-k_{s} \gamma\right) b_{2}\right|$ holds at $t=0.5$ and it is 0.0129 . Then, the following holds

$$
\max |s(t)| \leqslant 0.0728
$$

Note the agreement between the maximum of the piecewise sliding surface approximation and our result. The leading term in the later inequality is $\varepsilon \overline{\mathbb{U}}_{2}$ and it takes the value 0.0716 . Simulation results are depicted in Figure 4. 


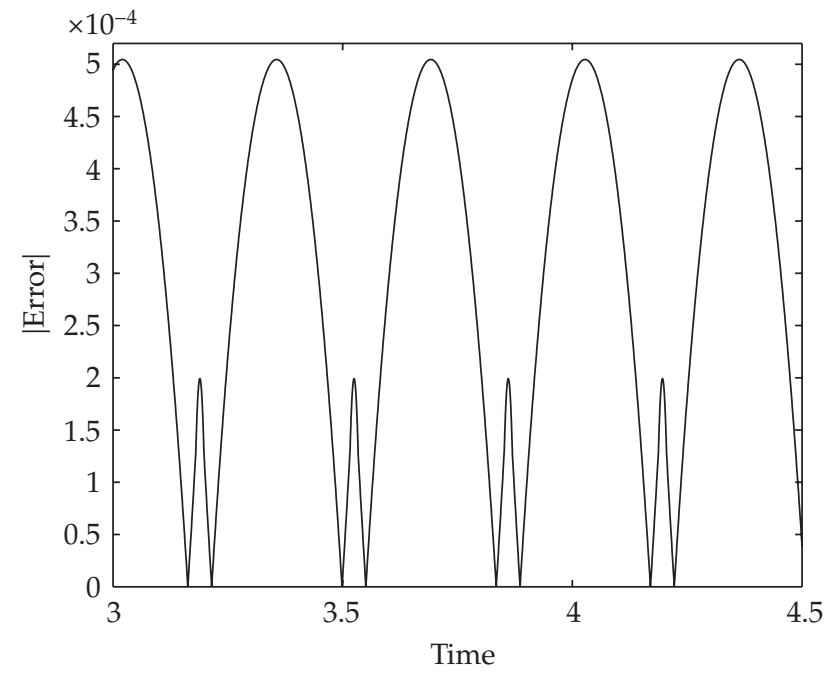

Figure 3: Behavior of the error in a sampling interval for CPWM scheme.

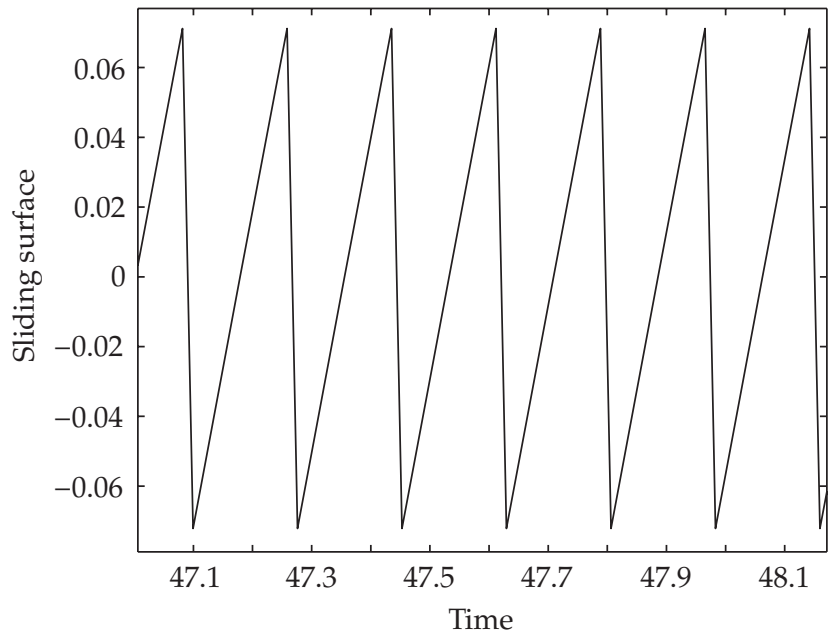

Figure 4: Behavior of the sliding surface in a sampling interval for CPWM scheme.

\subsection{LPWM}

In [12] it was proved that the limit of the stability is close to $k_{s}=0.182$. Values below this point lead the system to a chaotic behavior. Then, for obtaining a 1-periodic orbit, we use $k_{s}=0.7068$. With this value and the previous parameters, we obtain $\operatorname{det}\left(I-e^{\varepsilon \mathbf{A}}\right) \geqslant$ $0.0285,\left|e_{11}\right| \leqslant 1.2191,\left|e_{12}\right| \leqslant 0.2500,\left|e_{21}\right| \leqslant 0.3130$, and $\left|e_{22}\right| \leqslant 1.2191$. Therefore $I_{1} \leqslant$ $0.008, I_{2} \leqslant 0.0155,\left|m_{11}\right| \leqslant 0.2297,\left|m_{12}\right| \leqslant 0.2799,\left|m_{21}\right| \leqslant 0.3501$, and $\left|m_{22}\right| \leqslant 0.2915$. Thus, the bounds for $\mathbf{w}(0)$ and $\mathbf{w}(t)$ are

$$
\begin{array}{ll}
w_{1}(0) \leqslant 0.0012, & w_{2}(0) \leqslant 0.0014 \\
w_{1}(t) \leqslant 0.0019, & w_{2}(t) \leqslant 0.0022 .
\end{array}
$$




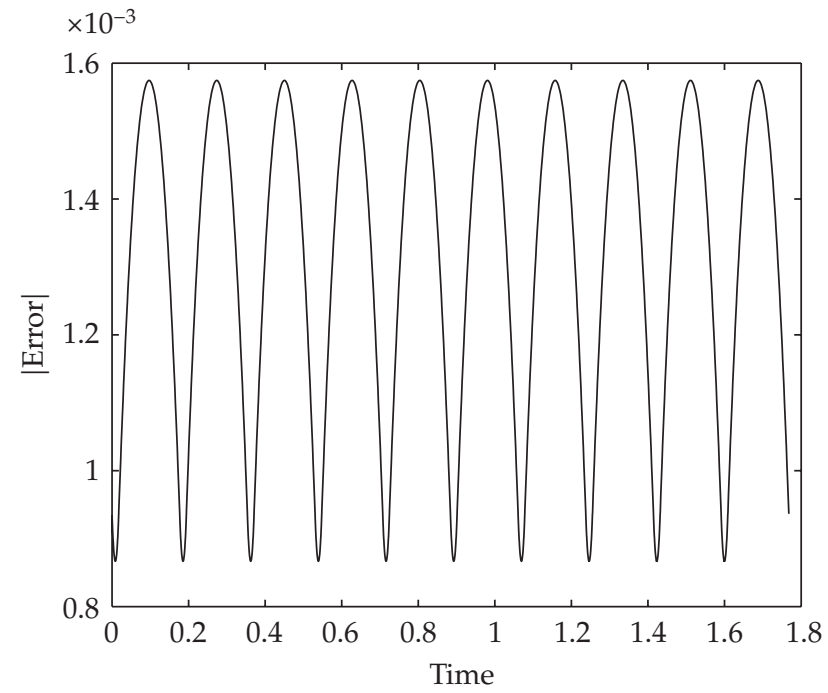

Figure 5: Behavior of the error in a sampling interval for LPWM scheme.

\subsubsection{Output error estimation}

Using (3.36), and computing $\mathbf{h}=\mathbf{A}^{-1} \mathbf{a}$, we obtain $\mathbf{h}=\left(\begin{array}{ll}0 & k_{s} \beta\end{array}\right)^{T}:=\left(\begin{array}{ll}0 & h_{2}\end{array}\right)^{T}$. As $\overline{\mathbb{U}}_{1}=0$, the error $e(t)$ fulfills

$$
e(t)=w_{1}(t)+\varepsilon^{2} \bar{u}_{1}
$$

The maximum value for excitation $\overline{\mathcal{U}}_{1}$ holds at $t=0.95$ and it is 0.0023 , hence,

$$
\max |e(t)| \leqslant 0.0019 \text {. }
$$

This is equivalent to a maximum error of $0.24 \%$ in steady state for a reference equal to 0.8 . The output error behavior is depicted in Figure 5. Simulations took 500 points per sampling period.

\subsubsection{Sliding surface error estimation}

In this case, we have

$$
s(t)=\varepsilon \overline{\mathbb{U}}_{2}+\varepsilon^{2} \overline{\mathcal{U}}_{2}+w_{2}(t)-\varepsilon h_{2} \leqslant \varepsilon\left|\overline{\mathbb{U}}_{2}-h_{2}\right|+\left|\varepsilon^{2} \overline{\mathcal{U}}_{2}\right|+\left|w_{2}(t)\right| .
$$

The maximum associated to $\left|\overline{\mathbb{U}}_{2}\right|$ holds at $t=0.9$ and it is 0.1272 , while the maximum of $\left|\overline{\mathcal{U}}_{2}\right|$ holds at $t=0.95$ and it is 0.0017 . In addition, $h_{2}=0.0636$; hence,

$$
\max |s(t)| \leqslant 0.0135 \text {. }
$$

Simulation results are depicted in Figure 6.

Previous results must be carefully applied. This is because $\mathbf{w}(0)$ is calculated assuming the existence and stability of 1-periodic orbit in (2.22) and all the procedure is based on the 


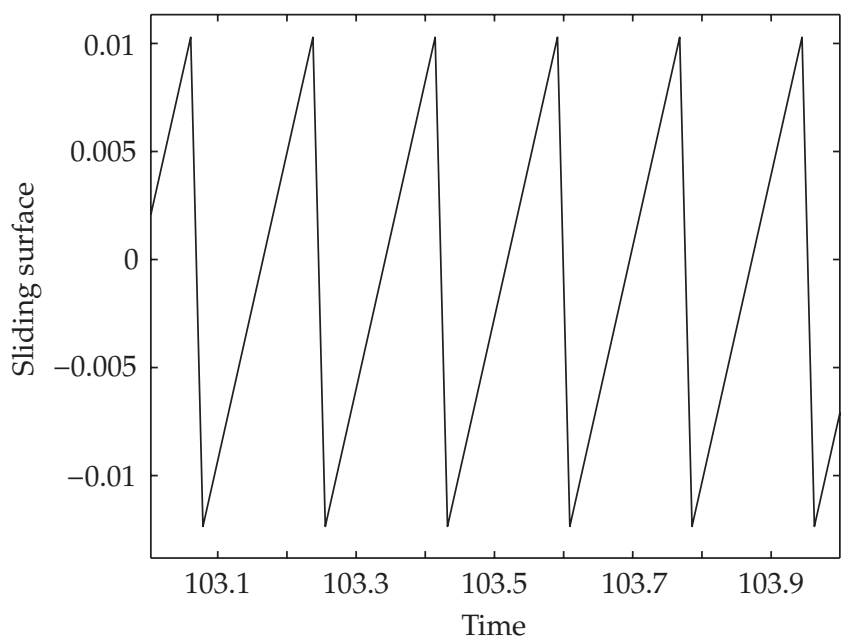

Figure 6: Behavior of the sliding surface in a sampling interval for LPWM scheme.

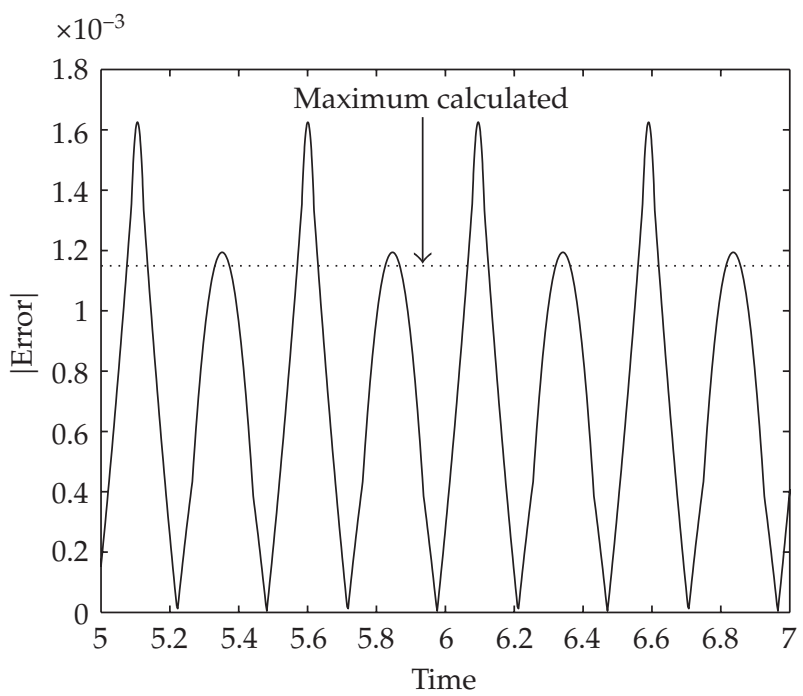

Figure 7: Behavior of the error for CPWM working in 2-periodic mode. In the same figure the bound calculated with the previous process is shown.

bound for $\mathbf{w}(0)$. Results obtained for other operation conditions may not agree. For example, the limit of the stability for this system is close to $k_{s}=3.24$ [12]. In particular, for $k_{s}=3.1$, the system works in 2-periodic mode. The results obtained when this technique is applied are shown below. In Figure 7, the behavior of the error is shown, and the maximum calculated with our approximations is presented too. Note that the predicted maximum does not agree with the numerical results. The reason is found in the duty cycle (see Figure 8). Now the system is 2-periodic and the steady state of the duty cycle is different from $d=0.9$. The same situation modifies the results obtained for the sliding surface (Figure 9). 


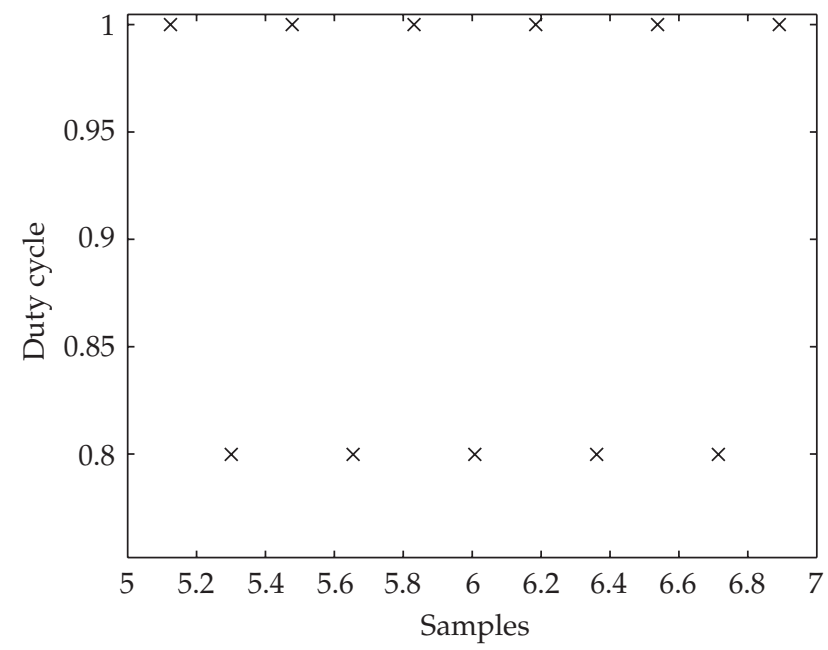

Figure 8: Duty cycle for CPWM working in 2-periodic mode. Note that the steady state is not $d=0.9$.

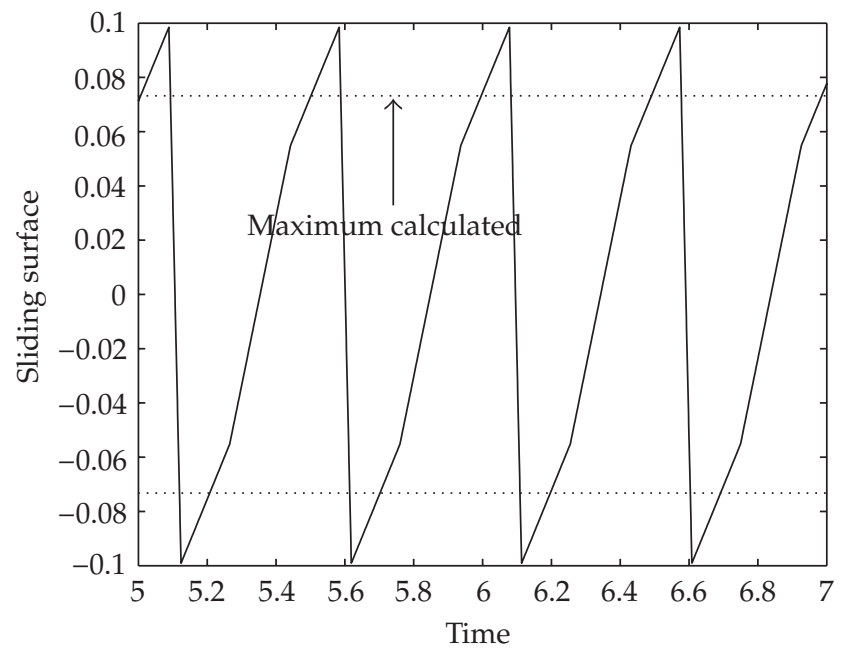

Figure 9: Behavior of the sliding surface for CPWM working in 2-periodic mode. In the same figure, the bound for the sliding surface.

Application of this technique in other operation points can lead to much higher or lower bounds compared to numerical results, and the approximation of the sliding surface to a piecewise straight line may not be valid.

\section{Generalizing the theory for second-order systems}

The objective of this section is to show that the procedure developed up to now is not only for a particular example, but is also correct for general second-order systems, as the following lemma shows. 
Lemma 5.1. Any stable second-order system with relative degree 2 and unitary gain can be put in the form of (2.2).

Proof. Any second linear order system with relative degree 2 and unitary gain is defined by the following transfer function

$$
Y(s)=\frac{\alpha_{1}}{s^{2}+\alpha_{2} s+\alpha_{1}} U(s)
$$

The system can be written as

$$
\left[\begin{array}{c}
\dot{x}_{1} \\
\dot{x}_{2}
\end{array}\right]=\left[\begin{array}{cc}
0 & 1 \\
-\alpha_{1} & -\alpha_{2}
\end{array}\right]\left[\begin{array}{l}
x_{1} \\
x_{2}
\end{array}\right]+\left[\begin{array}{c}
0 \\
\alpha_{1}
\end{array}\right] u, \quad y=\left[\begin{array}{ll}
1 & 0
\end{array}\right] \mathbf{x}
$$

The output corresponds to the first state of the new state vector. Doing $\tau=\left(1 / \sqrt{\alpha_{1}}\right) t$ with $\tau$ the current independent variable and $t$ the new independent variable, we have

$$
\frac{d x_{1}}{d t}=\frac{1}{\sqrt{\alpha_{1}}} x_{2} \triangleq w_{2}
$$

hence,

$$
\frac{d w_{2}}{d t}=\frac{1}{\alpha_{1}} \frac{d x_{2}}{d \tau}=-x_{1}-\frac{\alpha_{2}}{\sqrt{\alpha_{1}}} w_{2}+u
$$

Now, defining $w_{1}=x_{1}$, the dynamical system reads as

$$
\left[\begin{array}{c}
\dot{w}_{1} \\
\dot{w}_{2}
\end{array}\right]=\left[\begin{array}{cc}
0 & 1 \\
-1 & -\frac{\alpha_{2}}{\sqrt{\alpha_{1}}}
\end{array}\right]\left[\begin{array}{l}
w_{1} \\
w_{2}
\end{array}\right]+\left[\begin{array}{l}
0 \\
1
\end{array}\right] u, \quad y=\left[\begin{array}{ll}
1 & 0
\end{array}\right] \mathbf{w} .
$$

Finally, let us define $\gamma=\alpha_{2} / \sqrt{\alpha_{1}}, \mathbf{P}=\left(\begin{array}{ll}1 & 0 \\ \gamma & 1\end{array}\right)$ and $\mathbf{z}=\mathbf{P w}$, then (5.1) exactly matches to the system considered in (2.2). Even more, for the change of coordinates, it is obvious that $\alpha_{1} \neq 0$ guaranteeing the invertibility of the matrix A. Therefore, the methodology developed along the paper is a general tool.

\section{Conclusions}

From the previous sections we can conclude the following.

(i) Approximations done in [10-12] have been supported from averaging theory. In those papers, the sliding surface $s$ is assumed to be piecewise linear w.r.t. time. Our final expression reads as

$$
s(t)=\varepsilon \overline{\mathbb{U}}_{2}+\varepsilon^{2} \bar{U}_{2}+w_{2}(t)-\varepsilon h_{2}
$$

In this expression, the leading term is of order 1 . This is a straight line and it corresponds to the primitive of the input of the system. The other terms can be deleted, as being explained in Sections 4.1.2 and 4.2.2. 
(ii) Due to average theory, it has been analytically found that the maximum error is always less or equal to $0.3 \%$ for the parameter values in use. This confirms the effectiveness of the technique.

(iii) These results are strongly dependent on the constants values. A bad selection of the constants values can lead to wrong-predicted results (see Figures 7, 8, and 9). For example, the $k_{s}$ constant value considered in the LPWM case makes the system with CPWM unstable. For the general second-order system considered in the last section, the dynamics are defined by parameter $\gamma$. The results reported in the paper assume a 1-periodic orbit as steady-state dynamics.

(iv) The bounds depend explicitly on the reference value $\left(v_{\text {ref }}\right)$. As it can be seen, the error increases when reference decreases. These results are coherent with other numerical studies.

(v) A strong assumption of this procedure is given by the condition $\varepsilon\left|E^{*}\right|<1$ which is stated for avoiding zero division. However it is also clear that if this term is close to 1 , then the calculated bound will be very high. Thus, these considerations must be taken into account for applying these computed bounds.

(vi) CPWM and LPWM ZAD theory are generalized to any second-order system with unitary gain and relative degree 2 , and all the results obtained can be applied to another ZAD-controlled systems.

\section{References}

[1] C. Batlle, E. Fossas, and G. Olivar, "From Floquet exponents to control of chaos in piecewise linear systems," in Proceedings of IEEE International Symposium on Circuits and Systems (ISCAS '00), vol. 2, pp. 100-103, Geneva, Switzerland, May 2000.

[2] J. M. Ruiz, S. Lorenzo, I. Lobo, and J. Amigo, “Minimal UPS structure with sliding mode control and adaptive hysteresis band," in Proceedings of the 16th International Conference on Industrial Electronics Society (IECON '90), vol. 2, pp. 1063-1067, Pacific Grove, Calif, USA, November 1990.

[3] H. Pinheiro, A. S. Martins, and J. R. Pinheiro, "A sliding mode controller in single phase voltage source inverters," in Proceedings of the 20th International Conference on Industrial Electronics, Control and Instrumentation (IECON '94), vol. 1, pp. 394-398, Bologna, Italy, September 1994.

[4] Q. Yao and D. G. Holmes, "A simple, novel method for variable-hysteresis-band current control of a three phase inverter with constant switching frequency," in Proceedings of the 28th Industry Applications Society Annual Meeting (IAS '93), vol. 2, pp. 1122-1129, Toronto, Canada, October 1993.

[5] J. F. Silva and S. S. Paulo, "Fixed frequency sliding mode modulator for current mode PWM inverters," in Proceedings of the 24th IEEE Annual Power Electronics Specialists Conference (PESC '93), pp. 623-629, Seattle, Wash, USA, June 1993.

[6] L. Malesani, L. Rossetto, G. Spiazzi, and A. Zuccato, "An AC power supply with sliding-mode control," IEEE Industry Applications Magazine, vol. 2, no. 5, pp. 32-38, 1996.

[7] B. Nicolas, M. Fadel, and Y. Chéron, "Fixed-frequency sliding mode control of a single-phase voltage source inverter with input filter," in Proceedings of IEEE International Symposium on Industrial Electronics (ISIE '96), vol. 1, pp. 470-475, Warsaw, Poland, June 1996.

[8] L. J. Borle and C. V. Nayar, "Zero average current error controlled power flow for AC-DC power converters," IEEE Transactions on Power Electronics, vol. 10, no. 6, pp. 725-732, 1995.

[9] E. Fossas, R. Griño, and D. Biel, "Quasi-sliding control based on pulse width modulation, zero average and the $L_{2}$ norm," in Advances in Variable Structure System, Analysis, Integration and Applications, X. Yu and J.-X. Xu, Eds., pp. 335-344, World Scientific, River Edge, NJ, USA, 2001.

[10] D. B. Solé and E. F. Colet, "SMC applications in power electronics," in Variable Structure Systems: From Principles to Implementation, A. Sabanovič, L. Fridman, and S. Spurgeon, Eds., vol. 66 of IEE Control Engineering Series, pp. 265-293, Institution of Electrical Engineers (IEE), London, UK, 2004.

[11] R. R. Ramos, D. Biel, E. Fossas, and F. Guinjoan, "A fixed-frequency quasi-sliding control algorithm: application to power inverters design by means of FPGA implementation," IEEE Transactions on Power Electronics, vol. 18, no. 1, part 2, pp. 344-355, 2003. 
[12] F. Angulo, E. Fossas, and G. Olivar, "Transition from periodicity to chaos in a PWM-controlled buck converter with ZAD strategy," International Journal of Bifurcation and Chaos, vol. 15, no. 10, pp. 3245$3264,2005$.

[13] J. A. Sanders and F. Verhulst, Averaging Methods in Nonlinear Dynamical Systems, vol. 59 of Applied Mathematical Sciences, Springer, New York, NY, USA, 1985.

[14] H. K. Khalil, Nonlinear Systems, Prentice Hall, Upper Saddle River, NJ, USA, 2nd edition, 1996.

[15] M. Carpita, M. Marchesoni, M. Oberti, and L. Puguisi, "Power conditioning system using sliding model control," in Proceedings of the 19th IEEE Annual Power Electronics Specialists Conference (PESC'88), vol. 2, pp. 623-633, Kyoto, Japan, April 1988.

[16] F. Angulo, Análisis de la dinámica de convertidores electrónicos de potencia usando PWM basado en promediado cero de la dinámica del error(ZAD), Ph.D. thesis, Universidad Politécnica de Cataluña, Barcelona, Spain, July 2004.

[17] F. Angulo, C. Ocampo, G. Olivar, and R. Ramos, "Nonlinear and nonsmooth dynamics in a DC-DC Buck converter: two experimental set-ups," Nonlinear Dynamics, vol. 46, no. 3, pp. 239-257, 2006. 


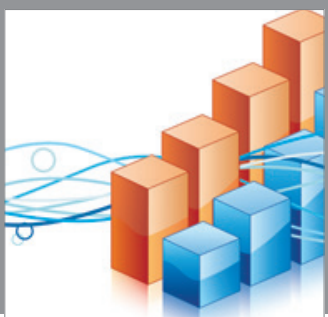

Advances in

Operations Research

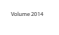

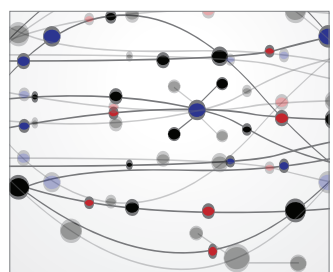

\section{The Scientific} World Journal
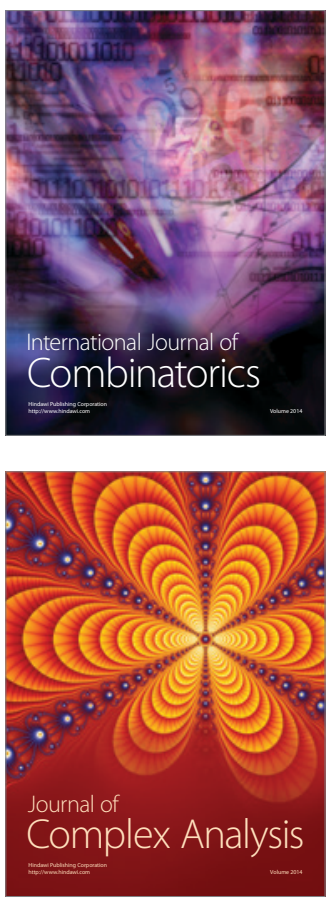

International Journal of

Mathematics and

Mathematical

Sciences
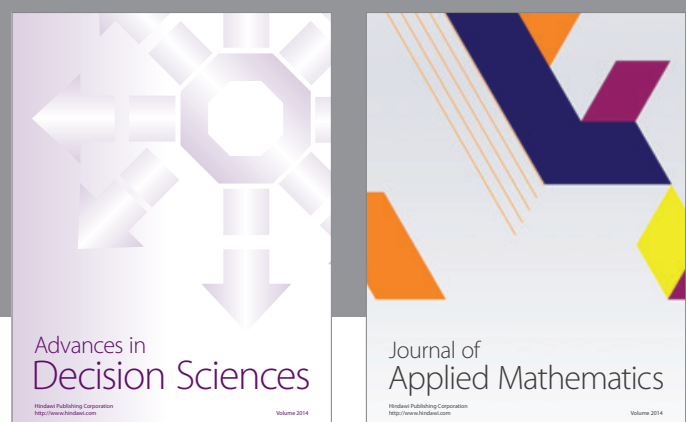

Journal of

Applied Mathematics
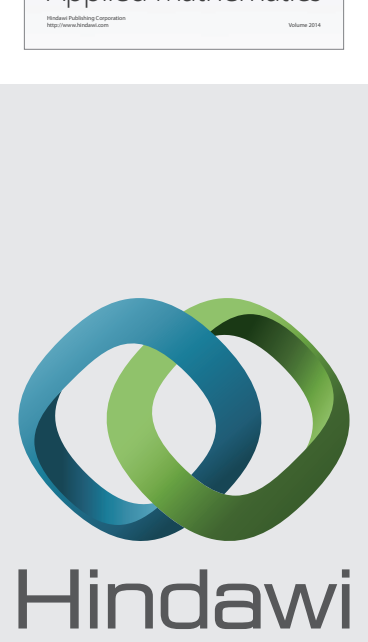

Submit your manuscripts at http://www.hindawi.com
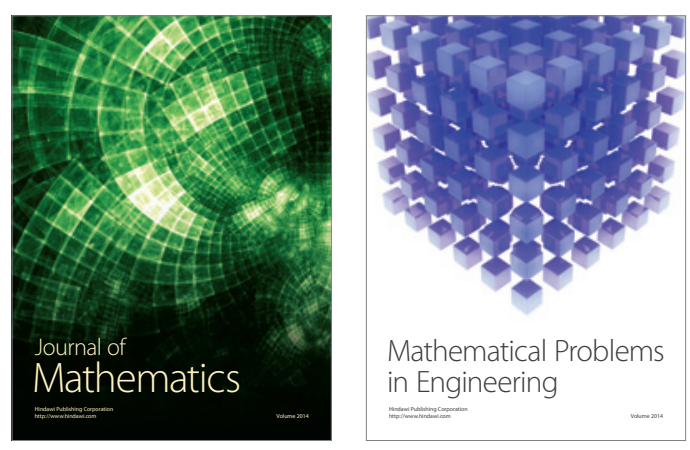

Mathematical Problems in Engineering
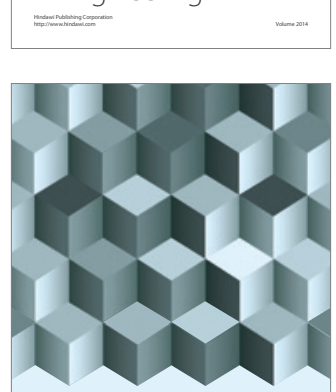

Journal of

Function Spaces
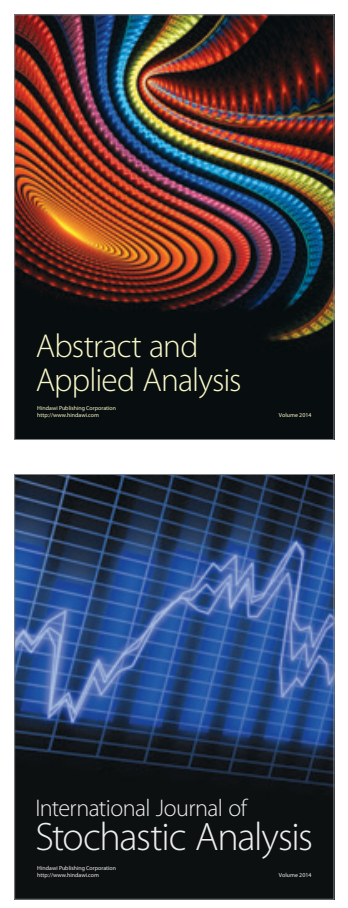

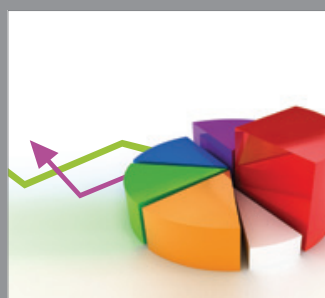

ournal of

Probability and Statistics

Promensencen
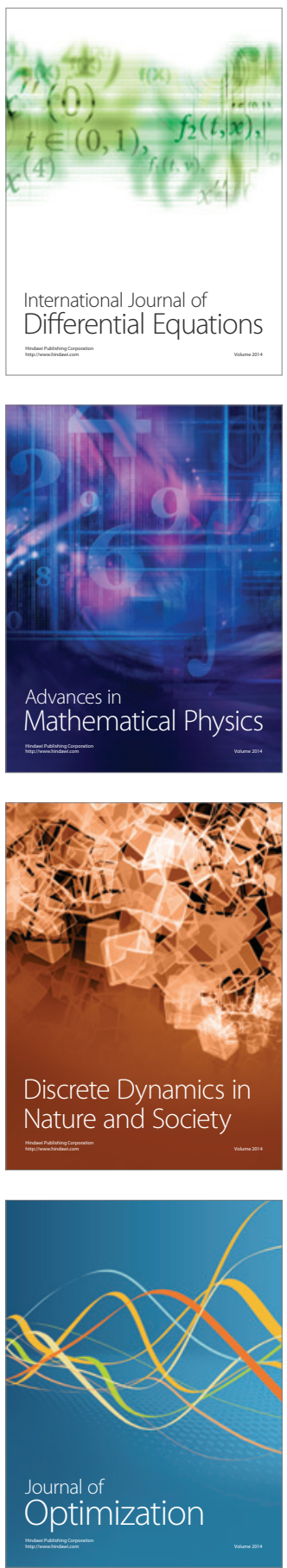